\title{
Neuroimaging of voice hearing in non-psychotic individuals: a mini review
}

\section{Kelly Maria Johanna Diederen*, Remko van Lutterveld and Iris E. C. Sommer}

Neuroscience Division, Rudolf Magnus Institute for Neuroscience, University Medical Center Utrecht, Utrecht, Netherlands

Edited by:

Kenneth Hugdahl, University of

Bergen, Norway

\section{Reviewed by:}

Nils I. Landro, University of Oslo, Norway

Vince D. Calhoun, University of

New Mexico, USA

*Correspondence:

Kelly Maria Johanna Diederen,

Department of Physiology,

Development and Neuroscience,

University of Cambridge, Downing

place, Cambridge CB2 3DY, UK.

e-mail:kmjd2@cam.ac.uk

\begin{abstract}
Auditory verbal hallucinations (AVH) or "voices" are a characteristic symptom of schizophrenia, but can also be observed in healthy individuals in the general population. As these non-psychotic individuals experience AVH in the absence of other psychiatric symptoms and medication-use they provide an excellent model to study $\mathrm{AVH}$ in isolation. Indeed a number of studies used this approach and investigated brain structure and function in non-psychotic individuals with $\mathrm{AVH}$. These studies showed that increased sensitivity of auditory areas to auditory stimulation and aberrant connectivity of language production and perception areas is associated with $\mathrm{AVH}$. This is in concordance with investigations that observed prominent activation of these areas during the state of $\mathrm{AVH}$. Moreover, while effortful attention appears not to be related to $\mathrm{AVH}$, individuals prone to hallucinate seem to have an enhanced attention bias to auditory stimuli which may stem from aberrant activation of the anterior cingulated regions. Furthermore, it was observed that decreased cerebral dominance for language and dopamine dysfunction, which are consistently found in schizophrenia, are most likely not specifically related to AVH as these abnormalities were absent in healthy voice hearers. Finally, specific aspects of AVH such as voluntary control may be related to the timing of the supplementary motor area and language areas in the experience of $\mathrm{AVH}$.
\end{abstract}

Keywords: auditory verbal hallucinations, non-psychotic individuals, psychosis, schizophrenia, fMRI, PET, EEG, DTI

\section{INTRODUCTION}

In contemporary Western societies auditory verbal hallucinations $(\mathrm{AVH})$ or "voices" are generally considered an aspect of disease (al-Issa, 1995). Indeed, AVH are frequently observed in individuals with a neurological, neurodegenerative, or psychiatric disorder (Aleman and Laroi, 2008). Moreover, these hallucinations are observed in $\sim 10-15 \%$ of healthy, i.e., non-psychotic, individuals in the general population (Tien, 1991; Sommer et al., 2008; Beavan et al., 2011). AVH are even more common during periods of partial wakefulness, i.e., when falling asleep (hypnagogic hallucinations) or during waking (hypnopompic hallucinations). $\mathrm{AVH}$ are, however, most common in schizophrenia, in which they occur with an average prevalence of 70\% (Sartorius et al., 1986).

While AVH are not associated with distress in non-psychotic individuals, they can seriously disrupt social functioning in psychotic patients (Nayani and David, 1996; Daalman et al., 2011). At present, the primary treatment for AVH in psychiatric patients consists of antipsychotic medication which is frequently combined with cognitive behavioral therapy. These hallucinations do, however, not respond to pharmaceutical intervention in $25-30 \%$ of patients, stressing the need for development of new treatment options (Shergill et al., 1998). This is, at present, hampered by the fact that the pathophysiology of AVH remains largely unknown.

Over the last decades a considerable number of studies aimed at elucidating the neurobiological mechanism of AVH in schizophrenia patients. Most of these can be divided into "symptomcapture" (a.k.a. "state" studies) on the one hand and "trait" investigations on the other hand (Kühn and Gallinat, 2010). The first group of studies investigated the neural signature of $\mathrm{AVH}$ and revealed that AVH-related brain activation can be observed in frontal and temporoparietal language areas as well as in the parahippocampal region (Jardri et al., 2010; Kühn and Gallinat, 2010). Although these studies provide a helpful start at understanding what happens in the brain during $\mathrm{AVH}$, they do not provide information about brain mechanisms predisposing a person to experience AVH. The latter can be provided by trait studies that focus on comparing brain activation between individuals with and without AVH. Previous studies showed that the propensity to hear voices may be associated with a number of factors including decreased left cerebral dominance for language (Levitan et al., 1999; Sommer et al., 2001; Weiss et al., 2006; Hugdahl et al., 2007), dysfunctional connectivity of frontal and temporoparietal language regions (Frith et al., 1995; Spence et al., 2000; Ford et al., 2007; Kühn and Gallinat, 2010), decreases in psychophysiological measures of effortful attention (Havermans et al., 1999; Turetsky et al., 2000), and dopamine dysfunction. It should, however, be noted that results are inconsistent.

While all of these factors may be involved in the genesis of $\mathrm{AVH}$, a key limitation is that most of these studies were conducted in patients with schizophrenia. Schizophrenia is a complex syndrome comprising positive, negative, and cognitive symptoms. In addition, schizophrenia patients typically use antipsychotic medication. As a result, observed deviations may not be specifically 
related to $\mathrm{AVH}$, but rather to another symptom or to general cognitive dysfunction observed in these patients. To elucidate if $\mathrm{AVH}$ are indeed specifically related to the aforementioned factors, they could be studied in (relative) isolation. Interestingly, previous studies have shown that AVH in the non-psychotic population frequently occur in the absence of other psychiatric symptoms and medication-use (Tien, 1991; Sommer et al., 2008; Beavan et al., 2011). These subjects thus provide an ideal opportunity to investigate a more isolated form of $\mathrm{AVH}$.

In this review, neuroimaging studies in non-psychotic individuals with AVH will be summarized. While different studies used different terminology for these individuals including non-clinical (Linden et al., 2011), non-psychotic (Diederen et al., 2010b, 2011; van Lutterveld et al., 2010; de Weijer et al., 2011), healthy (Howes et al., 2012), and hallucination-prone individuals (Lewis-Hanna et al., 2011), they will be termed non-psychotic individuals with $\mathrm{AVH}$ in this review. In the next section both state and trait studies will be described in detail. In addition to these studies which were conducted in individuals who present with non-evoked $\mathrm{AVH}$, two studies will be discussed in which AVH were evoked in hallucination-prone individuals using hypnosis or conditions of degraded stimulus information (Szechtman et al., 1998; Barkus et al., 2007).

\section{STATE STUDIES 1: NON-EVOKED HALLUCINATIONS PSYCHOTIC AND NON-PSYCHOTIC INDIVIDUALS}

A recent neuroimaging study into the state of $\mathrm{AVH}$ in nonpsychotic individuals with AVH compared brain activation during $\mathrm{AVH}$ between 21 non-psychotic and 21 psychotic subjects (Diederen et al., 2011). The rationale was that if AVH are caused by comparable mechanisms in both groups, one should observe a similar pattern of AVH-related brain activation. Indeed, the two groups displayed common areas of activation in the absence of any significant differences. These areas consisted of the bilateral inferior frontal gyri, insula, superior temporal gyri, supramarginal gyri and postcentral gyri, left precentral gyrus, inferior parietal lobule, superior temporal pole, and right cerebellum. Activation of these areas has been interpreted to reflect language and motor processes (perhaps as a result of balloon-squeezes used to indicate the AVH) in the experience of AVH.

\section{HALLUCINATIONS VS. IMAGERY}

More insight into the role of frontal and temporoparietal regions in the experience of $\mathrm{AVH}$ was provided by a recent study which compared AVH-related brain activation in seven non-psychotic individuals with AVH to imagery in 7 healthy subjects who did not present with AVH (Linden et al., 2011). This study revealed that both processes activated the supplementary motor area and bilateral frontotemporal language regions including the human voice area. This suggests that hallucinations as well as imagery are associated with an auditory percept. In addition, a difference in timing of brain regions was found for the two processes. While activity of the supplementary motor area preceded that of auditory areas during imagery, activation of these areas occurred instantaneously for AVH. The authors concluded that the different timing of the supplementary motor area is associated with the absence of control over the AVH in their group of individuals.

\section{STATE STUDIES 2: EVOKED HALLUCINATIONS HALLUCINATIONS, HEARING, AND IMAGERY DURING HYPNOSIS}

Imagery and $\mathrm{AVH}$ were also compared in an early study in which eight subjects hallucinated under hypnosis (Szechtman et al., 1998). In addition to AVH and imagery, brain activation during actual hearing was investigated in the same individuals as well as in six highly hypnotizable controls, without the ability to hallucinate under hypnosis, using PET. Both hallucinations and auditory stimulation, but not imagery, activated the right anterior cingulate area. Moreover, hallucination-related brain activation of this area correlated strongly with subjects' ratings of externality and clarity of the heard voice. Interestingly, hearing and imagery did not correlate with significant anterior cingulated activation in the control group of non-hallucinators from which the authors concluded that inappropriate activation of this region may lead self-generated thoughts to be experienced as external. It is, however, important to note that these results are not in line with the study by Linden et al. (2011) who found a similar pattern of activation during imagery and hallucinations. This might be related to the difference in evoked and non-evoked AVH.

\section{HALLUCINATIONS AND HEARING DURING STIMULUS DEGRADATION}

Using a different method to elicit hallucinations in hallucinationprone individuals Barkus et al. (2007) presented subjects with episodes of white noise during which a voice was presented part of the time. Subjects had to indicate if they had heard a voice during the noise resulting in a number of hits, but also "false alarms" which were used as a model for hallucinations. Eight of 68 subjects who reported hearing a voice when none was present repeated the task during functional imaging. Patterns of activation during false alarms showed activation in the right middle temporal gyrus, bilateral fusiform gyrus, and the right putamen. As this pattern of activation is rather similar to activation observed during $\mathrm{AVH}$ in patients the authors concluded that AVH in non-clinical samples appear to be mediated by similar patterns of cerebral activation as found during hallucinations in schizophrenia. Importantly, this is in line with the study by Diederen et al. (2011). For an overview of state studies on AVH see Table 1 .

\section{TRAIT STUDIES}

\section{PSYCHOPHYSIOLOGICAL PARAMETERS OF ATTENTION}

The first trait study in non-psychotic individuals with AVH investigated if $\mathrm{AVH}$ are specifically associated with dysfunction in psychophysiological parameters of attention (van Lutterveld et al., 2010). In this EEG study, three event related potentials (ERPs) were examined with an auditory oddball paradigm in 18 nonpsychotic individuals with AVH and 18 controls. While no significant differences were found for mismatch negativity, P300 amplitude was increased in the AVH group as compared to controls, reflecting superior effortful attention. A trend in the same direction was found for processing negativity. As the non-psychotic individuals with AVH showed increased rather than decreased psychophysiological measures of effortful attention, these results suggest that the decrease in EEG measures of effortful attention observed in schizophrenia patients is not related to the tendency to hallucinate. 
Table 1 | Symptome capture studies on AVH in non-psychotic individuals.

\begin{tabular}{|c|c|c|c|c|}
\hline Author & Subjects & Design & Method & Key neuroimaging results \\
\hline \multicolumn{5}{|l|}{ NON-EVOKED } \\
\hline $\begin{array}{l}\text { Linden et al. } \\
\text { (2011) }\end{array}$ & $\begin{array}{l}7 \text { Non-psychotic subjects } \\
\text { w. AVH } \\
7 \text { Non-hallucinator controls }\end{array}$ & $\begin{array}{l}\text { Brain activation during } \mathrm{AVH} \text { vs. } \\
\text { imagery }\end{array}$ & $\mathrm{fMRI}$ & $\begin{array}{l}\text { Similar act. AVH and imagery: } \uparrow \text { B voice area (STS), IFG, } \\
\text { cerebellum, planum temporale, prefrontal, inferior } \\
\text { parietal lobules, thalami, and L motor cortex } \\
\text { Group diff.: in controls(imagery) } \uparrow \text { SMA preceded } \uparrow \\
\text { auditory areas, in hallucinators activity occurred at same } \\
\text { time }\end{array}$ \\
\hline $\begin{array}{l}\text { Diederen et al. } \\
\text { (2011) }\end{array}$ & $\begin{array}{l}21 \text { Non-psychotic subjects } \\
\text { w. AVH } \\
21 \text { Psychotic patients with } \\
\text { AVH }\end{array}$ & Brain activation during $\mathrm{AVH}$ & $\mathrm{fMRI}$ & $\begin{array}{l}\text { Similar act.: } \uparrow \text { B IFG, insula, STG, SMG, postcentral gyri, } \\
\text { L precentral gyrus, inferior parietal lobule, superior } \\
\text { temporal pole, and right cerebellum } \\
\text { No sign. differences between groups }\end{array}$ \\
\hline \multicolumn{5}{|l|}{ EVOKED } \\
\hline $\begin{array}{l}\text { Szechtman et al. } \\
\text { (1998) }\end{array}$ & $\begin{array}{l}8 \text { Healthy volunteers w. } \\
\text { AVH under hypnosis } \\
6 \text { Healthy volunteers w.o. } \\
\text { AVH under hypnosis }\end{array}$ & $\begin{array}{l}\text { Brain activation during } \mathrm{AVH} \text {, } \\
\text { hearing, and imagining }\end{array}$ & PET & $\begin{array}{l}\text { AVH and Hearing (not imagining): } \uparrow R \text { anterior cingulate } \\
\text { hallucinating subjects } \\
\text { In non-hallucinating subject: no sign. activation in anteric } \\
\text { cingulate }\end{array}$ \\
\hline \multirow[t]{2}{*}{$\begin{array}{l}\text { Barkus et al. } \\
(2007)\end{array}$} & $\begin{array}{l}8 \text { High hallucination-prone } \\
\text { subjects }\end{array}$ & $\begin{array}{l}\text { AVH elicited using a signal } \\
\text { detection task } \\
\text { Brain activation during false } \\
\text { alarms (AVH), rejections (voice }\end{array}$ & $\mathrm{fMRI}$ & $\begin{array}{l}\text { False alarms - correct rejections: } \uparrow \mathrm{R} \text { MTG, B fusiform } \\
\text { gyrus, and R putamen }\end{array}$ \\
\hline & & $\begin{array}{l}\text { present but not detected), and } \\
\text { hits (voice correctly detected) }\end{array}$ & & $\begin{array}{l}\text { False alarms-hits: } \uparrow \text { R SFG, MFG, L cingulate gyrus, } \\
\text { MTG, cerebellum, and B STG }\end{array}$ \\
\hline
\end{tabular}

W., with; w.o., without; AVH, auditory verbal hallucinations; fMRl; functional magnetic resonance imaging; $P E T$, positron emission tomography; $B$, bilateral; $L$, left; $R$, right; STS, superior temporal sulcus; IFG, inferior frontal gyrus; SMA, supplementary motor area; STG, superior temporal gyrus; SMG, supramarginal gyrus; MTG, middle temporal gyrus; SFG, superior frontal gyrus; MFG, middle frontal gyrus.

\section{LANGUAGE LATERALIZATION}

An influential theory on $\mathrm{AVH}$ poses that $\mathrm{AVH}$ result from decreased left cerebral dominance for language, i.e., language lateralization (Sommer and Diederen, 2009). To test this hypothesis a second trait study compared language lateralization between 35 non-psychotic individuals with AVH and 35 psychotic patients as well as 35 healthy control subjects (Diederen et al., 2010b). While the patients displayed decreased language lateralization, this could not be observed in the non-psychotic individuals with AVH. It is therefore not considered likely that these hallucinations result from decreased cerebral dominance for language.

\section{FRONTOTEMPORAL CONNECTIVITY}

The most influential contemporary model poses that AVH occur due to a failure to recognize self-generated inner speech (Frith et al., 1995; Spence et al., 2000; Ford et al., 2007). This is hypothesized to result from dysfunctional integration of frontotemporal language production and perception areas which are connected via the arcuate fasciculus. To address this hypothesis, a recent study compared tract integrity of the arcuate fasciculus and three other white matter tracts between 35 non-psychotic individuals with AVH, 35 schizophrenia patients with $\mathrm{AVH}$, and 36 controls (de Weijer et al., 2011). While patients showed abnormalities in multiple white matter tracts, the healthy individuals with AVH only showed microstructural aberrations in the arcuate fasciculus, which is in line with the inner speech model of AVH.

\section{AUDITORY ATTENTION AND STIMULATION}

With a primary focus on auditory processes Lewis-Hanna et al. (2011) investigated 12 individuals who were prone to sleep-related (i.e., hypnagogic and hypnopompic) hallucinations. The authors examined speech-evoked brain activation as well as modulation of brain activation by auditory attention using fMRI. Hallucinating individuals demonstrated greater speech-evoked activation in the left supramarginal gyrus compared to a control group of similar sample size. In addition, directing attention towards the auditory modality was associated with greater activation of the anterior cingulate gyrus in the hallucinator group. The authors concluded that hallucination-proneness is associated with increased sensitivity of auditory areas to auditory stimulation, which might arise due to an enhanced attentional bias from the anterior cingulate gyrus.

\section{DOPAMINE FUNCTION}

Another process associated with the origin of AVH is dopaminergic dysfunction which has been frequently observed in psychotic patients (Laruelle and Abi-Dargham, 1999; Howes et al., 2007). Thus far, it is not clear if this dysfunction is related to psychosis in general or to a specific symptom. To elucidate if such a specific association with AVH exists, Howes et al. (2012) compared dopamine synthesis capacity in 16 non-psychotic individuals with AVH to 16 controls and showed that no significant difference could be observed between the groups (Howes et al., 2012). The authors concluded from this that altered dopamine synthesis capacity is 
Table 2 | Trait studies on AVH in non-psychotic individuals.

\begin{tabular}{|c|c|c|c|c|}
\hline Author & Subjects & Design & Method & Key neuroimaging results \\
\hline $\begin{array}{l}\text { van Lutterveld } \\
\text { et al. (2010) }\end{array}$ & $\begin{array}{l}18 \text { Non-psychotic subjects w. AVH } \\
18 \text { Non-hallucinator controls }\end{array}$ & $\begin{array}{l}\text { P300 waveforms, PN, and MMN } \\
\text { with an auditory oddball paradigm }\end{array}$ & $\begin{array}{l}\text { EEG- } \\
\text { ERP }\end{array}$ & $\begin{array}{l}\uparrow \text { P300 amplitude in non-psychotic subjects } \\
\uparrow \mathrm{PN} \text { amplitude (trend-level) in } \\
\text { non-psychotic subjects } \\
\text { No sign. group difference MMN }\end{array}$ \\
\hline $\begin{array}{l}\text { Diederen et al. } \\
(2010 b)\end{array}$ & $\begin{array}{l}35 \text { Non-psychotic subjects w. AVH } \\
35 \text { Psychotic patients w. AVH } \\
35 \text { Non-hallucinator controls }\end{array}$ & Covert verbal fluency task & $\mathrm{fMRI}$ & $\begin{array}{l}\downarrow \text { Language lateralization in patients, not } \\
\text { non-psychotic subjects w. AVH }\end{array}$ \\
\hline $\begin{array}{l}\text { de Weijer et al. } \\
\text { (2011) }\end{array}$ & $\begin{array}{l}35 \text { Non-psychotic subjects w. AVH } \\
35 \text { Schizophrenia patients w. AVH } \\
36 \text { Non-hallucinator controls }\end{array}$ & $\begin{array}{l}\text { Tract integrity of the AF and } \\
\text { control tracts (CST, CGL, and UF) }\end{array}$ & $\begin{array}{l}\text { DTI and } \\
\mathrm{MTI}\end{array}$ & $\begin{array}{l}\uparrow M T R \text { in } L A F \text { in non-psychotic individuals } \\
\text { and patients w. AVH } \\
\uparrow M T R R A F \text { in patients } \\
\downarrow \text { FA in L AF, R CST, and B UF in patients }\end{array}$ \\
\hline $\begin{array}{l}\text { Lewis-Hanna } \\
\text { et al. (2011) }\end{array}$ & $\begin{array}{l}12 \text { Non-pyschotic subjects w. } \\
\text { auditory HG/HP hallucinations } \\
12 \text { Non-hallucinator controls }\end{array}$ & $\begin{array}{l}\text { Speech-evoked brain activation } \\
\text { and selective attention paradigm } \\
\text { (auditory/visual) }\end{array}$ & $f M R I$ & $\begin{array}{l}\uparrow \text { Speech-evoked act. L SMG in } \\
\text { hallucinating group } \\
\uparrow \text { Anterior cingulate activity when directing } \\
\text { attention to auditory (vs. visual) modality in } \\
\text { hallucinating subjects }\end{array}$ \\
\hline $\begin{array}{l}\text { Howes et al. } \\
\text { (2012) }\end{array}$ & $\begin{array}{l}16 \text { Non-subjects w. AVH } \\
16 \text { Non-hallucinator controls }\end{array}$ & [18F]-DOPA PET & PET & $\begin{array}{l}\text { No significant group difference in striatal } \\
\text { dopamine synthesis capacity }\end{array}$ \\
\hline
\end{tabular}

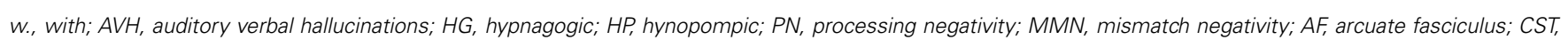

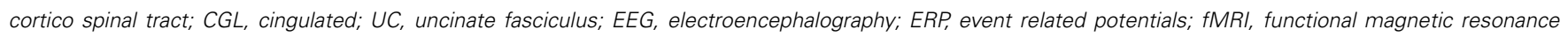
imaging; DTI, diffusion tensor imaging; MTI, magnetic transfer imaging; PET, positron emission tomography; $B$, bilateral; L, left; $R$, right; SMG, supramarginal gyrus.

unlikely to underlie (sub-clinical) hallucinations. For an overview of trait studies on AVH see Table 2.

\section{DISCUSSION}

In summary, these studies show that while decreased cerebral dominance for language and dopamine dysfunction are presumably not specifically related to AVH, increased sensitivity of auditory areas to auditory stimulation and aberrant connectivity of language production and perception areas is particularly associated with these hallucinations. This is in concordance with state studies which observed prominent activation of these areas during the state of AVH (Jardri et al., 2010; Kühn and Gallinat, 2010). Moreover, while effortful attention appears not to be related to AVH, individuals prone to hallucinate seem to have an enhanced attentional bias to auditory stimuli which may stem from aberrant activation of the anterior cingulated regions. Furthermore, specific aspects of AVH such as voluntary control may be related to the timing of the supplementary motor area and language areas in the experience of AVH.

In addition, these studies imply that investigating nonpsychotic individuals with AVH provides an excellent model to disentangle which dysfunctions may be specifically related to these hallucinations. Future studies should therefore focus on investigating additional processes which have been associated with $\mathrm{AVH}$, including for instance the role of top-down processes and anatomical integrity of language regions. A second aim of future studies should consist of investigating brain activation preceding AVH as such studies in psychotic patients showed that AVH are most likely instantiated by memory retrieval (Hoffman et al.,
2008, 2011; Diederen et al., 2010a). Finally, future studies could elucidate how specific AVH-characteristics are associated with brain activation. As psychotic and non-psychotic individuals display both differences and similarities with respect to specific AVHcharacteristics it would be ideal to combine these groups in such a study (Daalman et al., 2011).

\section{METHODOLOGICAL CONSIDERATIONS}

These studies should be interpreted with caution as individuals with both evoked and non-evoked AVH participating in most of these studies were highly selected. Furthermore, a limitation is that most of the state studies included only a small number of subjects, i.e., typically less than ten per group. In addition, one may wonder if these individuals should indeed be considered non-psychotic. If strict Diagnostic and Statistical Manual of Mental Disorders (DSM) IV criteria for axis I were applied, all subjects with non-evoked and non-sleep-related $\mathrm{AVH}$ would meet criteria for psychosis not otherwise specified (NOS) as all participants met the criterion persistent hallucinations, which in itself is sufficient for this classification. However, the DSM general terms state that a person has to be bothered by his symptoms and/or dysfunction on social, psychological, and professional domains should be present in order to make a diagnosis. The fact that the hallucinating subjects participating in the studies described showed no social, affective, or professional dysfunction, were not bothered by the AVH and were not in need of treatment, indicates that the diagnoses psychosis NOS is clinically inappropriate (Sommer et al., 2008). 


\section{REFERENCES}

Aleman, A., and Laroi, F. (2008). Hallucinations: The Science of Idiosyncratic Perception. Washington, DC: American Psychological Association.

al-Issa, I. (1995). The illusion of reality or the reality of illusion. Hallucinations and culture. Br. J. Psychiatry 166, 368-373.

Barkus, E. J., Stirling Hopkins, R., McKie, S., and Lewis, S. (2007). Cognitive and neural processes in nonclinical auditory hallucinations. $\mathrm{Br}$. J. Psychiatry 191, s76-s81.

Beavan, V., Read, J., and Cartwright, C. (2011). The prevalence of voicehearers in the general population: a literature review. J. Ment. Health 20, 281-292.

Daalman, K., Boks, M. P., Diederen, K. M., de Weijer, A. D., Blom, J. D., Kahn, R. S., and Sommer, I. E. (2011). The same or different? A phenomenological comparison of auditory verbal hallucinations in healthy and psychotic individuals. J. Clin. Psychiatry 72, 320-325.

de Weijer, A. D., Neggers, S. F. W., Diederen, K. M., Mandl, R. C., Kahn, R. S., Hulshoff Pol, H. E., and Sommer, I. E. (2011). Aberrations in the arcuate fasciculus are associated with auditory verbal hallucinations in psychotic and in non-psychotic individuals. Hum. Brain Mapp. 130, 68-77.

Diederen, K. M., Neggers, S. F., Daalman, K., Blom, J. D., Goekoop, R., Kahn, R. S., and Sommer, I. E. (2010a). Deactivation of the parahippocampal gyrus preceding auditory hallucinations in schizophrenia. Am. J. Psychiatry 167, 427-435.

Diederen, K. M. J., De Weijer, A. D., Daalman, K., Blom, J. D., Neggers, S. F. W., Kahn, R. S., and Sommer, I. E. C. (2010b). Decreased language lateralization is characteristic of psychosis, not auditory hallucinations. Brain 133, 3734-3744.

Diederen, K. M., Daalman, J. K., de Weijer, A. D., Neggers, S. F., van Gastel, W., Blom, J. D., Kahn, R. S., Sommer, I. E., and Bulletin, S. (2011). Auditory hallucinations elicit similar brain activation in psychotic and nonpsychotic individuals. Schizophr. Bull. doi: 10.1093/schbul/ sbr033

Ford, M. J., Roach, B. J., Faustman, W. O., and Mathalon, D. H. (2007). Synch Before You Speak: Auditory Hallucinations in Schizophrenia. Arlington, VA: American Psychiatric Association.
Frith, C. D., Friston, K. J., Herold, S., Silbersweig, D., Fletcher, P., Cahill, C., Dolan, R. J., Frackowiak, R. S., and Liddle, P. F. (1995). Regional brain activity in chronic schizophrenic patients during the performance of a verbal fluency task. $B r$. J. Psychiatry 167, 343-349.

Havermans, R., Honig, A., Vuurman, E. F., Krabbendam, L., Wilmink, J., Lamers, T., Verheecke, C. J., Jolles, J., Romme, M. A., and van Praag, H. M. (1999). A controlled study of temporal lobe structure volumes and $\mathrm{P} 300$ responses in schizophrenic patients with persistent auditory hallucinations. Schizophr. Res. 38, 151-158.

Hoffman, R. E., Anderson, A. W., Varanko, M., Gore, J. C., and Hampson, M. (2008). Time course of regional brain activation associated with onset of auditory/verbal hallucinations. Br. J. Psychiatry 193, 424-425.

Hoffman, R. E., Pittman, B., Constable, R. T., Bhagwagar, Z., and Hampson, M. (2011). Time course of regional brain activity accompanying auditory verbal hallucinations in schizophrenia. Br. J. Psychiatry 198, 277-283.

Howes, O. D., Montgomery, A. J., Asselin, M. C., Murray, R. M., Grasby, P. M., and McGuire, P. K. (2007). Molecular imaging studies of the striatal dopaminergic system in psychosis and predictions for the prodromal phase of psychosis. $\mathrm{Br}$. J. Psychiatry 191, s13-s18.

Howes, O. D., Shotbolt, P., Bloomfield, M., Daalman, K., Demjaha, A., Diederen, K. M. J., Ibrahim, K., Kim, E., McGuire, P., Kahn, R. S., and Sommer, I. E. (2012). Dopaminergic function in the psychosis spectrum: an [18F]-DOPA Imaging Study in Healthy Individuals with Auditory Hallucinations. Schizophr. Bull. doi: 10.1093/schbul/sbr195

Hugdahl, K., Loberg, E. M., Specht, K., Steen, V. M., van Wageningen, H., and Jørgensen, H. A. (2007). Auditory hallucinations in schizophrenia: the role of cognitive, brain structural and genetic disturbances in the left temporal lobe. Front. Hum. Neurosci. 1:6. doi:10.3389/neuro.09.006.2007

Jardri, R., Pouchet, A., Pins, D., and Thomas, P. (2010). Cortical activations during auditory verbal hallucinations in schizophrenia: a coordinate-based meta-analysis. Am. J. Psychiatry 168, 73-81.

Kühn, S., and Gallinat, J. (2010). Quantitative meta-analysis on state and trait aspects of auditory verbal hallucinations in schizophrenia. Schizophr. Bull. doi: 10.1093/schbul/sbq152

Laruelle, M., and Abi-Dargham, A. (1999). Dopamine as the wind of the psychotic fire: new evidence from brain imaging studies. J. Psychopharmacol. 13, 358-371.

Levitan, C., Ward, P. B., and Catts, S. V. (1999). Superior temporal gyral volumes and laterality correlates of auditory hallucinations in schizophrenia. Biol. Psychiatry 46, 955-962.

Lewis-Hanna, L. L., Hunter, M. D., Farrow, T. F., Wilkinson, I. D., and Woodruff, P. W. (2011). Enhanced cortical effects of auditory stimulation and auditory attention in healthy individuals prone to auditory hallucinations during partial wakefulness. Neuroimage 57, 1154-1161.

Linden, D. E. J., Thornton, K., Kuswanto, C. N., Johnston, S. J., van de Ven, V., and Jackson, M. C. (2011). The brain's voices: comparing nonclinical auditory hallucinations and imagery. Cereb. Cortex 21, 330-337.

Nayani, T. H., and David, A. S. (1996). The auditory hallucination: a phenomenological survey. Psychol. Med. 26, 177-189.

Sartorius, N., Jablensky, A., Korten, A., Ernberg, G., Anker, M., Cooper, J. E., and Day, R. (1986). Early manifestations and first-contact incidence of schizophrenia in different cultures. A preliminary report on the initial evaluation phase of the WHO Collaborative Study on determinants of outcome of severe mental disorders. Psychol. Med. 16, 909-928.

Shergill, S. S., Murray, R. M., and McGuire, P. K. (1998). Auditory hallucinations: a review of psychological treatments. Schizophr. Res. 32, 137-150.

Sommer, I. E., Daalman, K., Rietkerk, T., Diederen, K. M., Bakker, S., Wijkstra, J., and Boks, M. P. (2008). Healthy individuals with auditory verbal hallucinations; who are they? Psychiatric assessments of a selected sample of 103 subjects. Schizophr. Bull. 36, 633-641.

Sommer, I. E., and Diederen, K. M. (2009). Language production in the non-dominant hemisphere as a potential source of auditory verbal hallucinations. Brain 132, e124.

Sommer, I. E., Ramsey, N. F., and Kahn, R. S. (2001). Language lateralization in schizophrenia, an fMRI study. Schizophr. Res. 52, 57-67.
Spence, S. A., Grasby, P. M., Liddle, P. F., Stefan, M. D., Sharma, T., Murray, R. M., Hellewell, J. S. E., Friston, K. J., Hirsch, S. R., and Deakin, J. F. W. (2000). Functional anatomy of verbal fluency in people with schizophrenia and those at genetic risk. Focal dysfunction and distributed disconnectivity reappraised. $\mathrm{Br}$. J. Psychiatry 176, 52-60.

Szechtman, H., Woody, E., Bowers, K. S., and Nahmias, C. (1998). Where the imaginal appears real: a positron emission tomography study of auditory hallucinations. Proc. Natl. Acad. Sci. U.S.A. 95, 1956-1960.

Tien, A. Y. (1991). Distributions of hallucinations in the population. Soc. Psychiatry Psychiatr. Epidemiol. 26, 287-292.

Turetsky, B. I., Cannon, T. D., and Gur, R. E. (2000). P300 subcomponent abnormalities in schizophrenia: III. Deficits in unaffected siblings of schizophrenic probands. Biol. Psychiatry 47, 380-390.

van Lutterveld, R., Oranje, B., Kemner, C., Abramovic, L., Willems, A. E., Boks, M. P., Glenthøj, B. Y., Kahn, R. S., and Sommer, I. E. (2010). Increased psychophysiological parameters of attention in nonpsychotic individuals with auditory verbal hallucinations. Schizophr. Res. 121, 153-159.

Weiss, E. M., Hofer, A., Golaszewski, S., Siedentopf, C., Felber, S., and Fleischhacker, W. W. (2006). Language lateralization in unmedicated patients during an acute episode of schizophrenia: a functional MRI study. Psychiatry Res. 146, 185-190.

Conflict of Interest Statement: The authors declare that the research was conducted in the absence of any commercial or financial relationships that could be construed as a potential conflict of interest.

Received: 31 December 2011; accepted: 12 April 2012; published online: 09 May 2012.

Citation: Diederen KMJ, van Lutterveld $R$ and Sommer IEC (2012) Neuroimaging of voice hearing in nonpsychotic individuals: a mini review. Front. Hum. Neurosci. 6:111. doi: 10.3389/fnhum.2012.00111

Copyright (c) 2012 Diederen, van Lutterveld and Sommer. This is an open-access article distributed under the terms of the Creative Commons Attribution Non Commercial License, which permits noncommercial use, distribution, and reproduction in other forums, provided the original authors and source are credited. 